\title{
Unsäglicher Körpermief Mann, stinkt der nach Fisch!
}

\begin{abstract}
„Was liegt denn hier? Ein Mann oder ein Fisch? Tot oder lebendig?", lässt William Shakespeare einen Protagonisten in seinem Stück "der Sturm" sagen. Für einen 45-jährigen Mann war dieses Zitat leider kein Theater, sondern bedrückende Realität.
\end{abstract}

Der Mann suchte bei Prof. Dr. Prabath WB Nanayakkara und seinen Kollegen an der Medizinischen Klinik der Universität Amsterdam Hilfe, da er unglaublich stark nach Fisch roch - und das bildete er sich nicht nur ein, der Geruch war auch für die Umwelt zu riechen. Seine Frau hatte festgestellt, dass Schweiß, Urin, Sperma und Speichel allesamt diesen unangenehmen Fischgeruch hatten, der sich abhängig von der Ernährung noch verschlimmerte. Parfüm, literweise angewendet, sollte das ungewöhnliche Aroma kaschieren. Auch seiner beruflichen Laufbahn kam der Fischgeruch in die Quere: Man bot ihm einen neuen Job an, den er aber nicht annahm - er hätte mit anderen Kollegen in einem Büro sitzen müssen! Dementsprechend wundert es nicht, dass der Mann psychisch durch Depressionen und Angstattacken stark angeschlagen war, sein Selbstwertgefühl ging gegen Null.

\section{Spezieller Enzymmangel}

Eine Internetrecherche brachte die Klinikärzte zu der Diagnose Trimethylaminurie. Dabei handelt es sich um eine Stoffwechselkrankeit, bei der es an einem speziellen Leberenzym mangelt, wodurch es durch fehlenden Abbau zu einer Anreicherung von Trimethylamin im Körper und die Ausscheidung über Schweiß, Urin und Co. kommt. Das Amin ist ein
Als ob sie mit einem ranzigen Fisch im Bett läge ... stark riechender Metabolit des Cholinstoffwechsels und wird normalerweise zu dem geruchlosen Trimethylamin-NOxid (TMAO) abegbaut. Bekannt ist der Geruch vom Fisch, da Seefisch viel TMAO enthält und dieses bei Lagerung wieder in das Amin umgewandelt wird - somit ist der Geruch ein untrügliches Zeichen, dass der Fisch nicht mehr ganz frisch ist.

\section{Strikte Diåt ist angesagt}

Grund für den Enzymmangel ist eine Genmutation, die die Ärzte auch tatsächlich bei ihrem Patienten fanden. Problem erkannt, Problem gebannt, hieß es danach: Der Mann bekam eine strikte Diät verordnet, die möglichst arm an Cholin war. Das heißt: kein Fisch, keine Eier, keine Hülsenfrüchte, keine Innereien. Mit dieser Ernäh- rungsweise nahm der Geruch deutlich ab und der Mann war mit sich und seinem Leben wieder mehr im Reinen.

Dass dieses Fischgeruch-Syndrom schon länger bekannt ist, verrät der Blick in die Literatur - wie sonst wäre Shakespeare auf die Idee gekommen, eine Figur aus seinem Theaterstück, den Sklaven Caliban, an dieser Erkrankung leiden zu lassen. Caliban muss sich folgende Beschreibung seiner selbst anhören: „Ein Fisch, er riecht wie ein Fisch, 's ist ein recht ranziger und fischiger Geruch, so 'ne Art Laberdan*, nicht von dem frischesten, ein seltsamer Fisch!“

(Swanett Koops)

Lancet 2010, 376:1710

* Laberdan: Direkt am Fangort in Salzlake eingelegter und in Holzfässern geschichteter Kabeljau oder Seehecht

\section{Langzeitprotektion gegen Darmkrebs Reichen 75 mg Aspirin?}

\section{Ab welcher Dosis wirkt Aspirin pro- tektiv auf den Darm? Dieser Frage gingen Forscher aus $\mathrm{Oxford}$ nach und wurden in fünf randomisierten Stu- dien fündig.}

Das hohe Dosen Aspirin (500 mg/Tag) das Darmkrebsrisiko reduzieren, ist bekannt. Eine aktuelle Studie belegt jetzt, dass auch geringere Mengen eine protektive Wirkung zeigen. Aus der Nachbeobachtung von vier randomisierten Studien mit 14.033 Patien- ten geht hervor, dass Aspirin bereits ab einer Dosis von $75 \mathrm{mg} / \mathrm{Tag}$ vor allem das Risiko eines Karzinoms im proximalen Kolon mindert. Entscheidend ist dabei die regelmäßige Einnahme über mindestens fünf Jahre. Auch das Risiko eines rektalen Karzinoms wird dadurch verringert.

Die fünfte Studie weist allerdings auf eine geringere Darmkrebssterblichkeit bei einer Dosis von $283 \mathrm{mg} / \mathrm{Tag}$ gegenüber $30 \mathrm{mg} /$ Tag hin.

(ch)

Rothwell PM et al, Lancet 2010, 376:1741 